\title{
Circ_0020123 regulates autophagy, glycolysis, and malignancy by upregulating IRF4 through eliminating miR-193a-3p-mediated suppression of IRF4 in non-small cell lung cancer
}

\author{
Xing-Ping YANG ${ }^{1, *}$, Yu-Zhen ZHENG ${ }^{1, *}$, Jian TAN ${ }^{1}$, Ren-Jiang TIAN², Piao SHEN ${ }^{2}$, Wei-Jie CAI ${ }^{1}$, Hong-Ying LIAO ${ }^{1, *}$ \\ ${ }^{1}$ Department of Thoracic Surgery, Thoracic Cancer Center, The Sixth Affiliated Hospital of Sun Yat-sen University, Guangzhou, China; ${ }^{2}$ Depart- \\ ment of Thoracic Surgery, Affiliated Cancer Hospital and Institute of Guangzhou Medical University, Guangzhou, China
}

${ }^{*}$ Correspondence: liaohy2@mail.sysu.edu.cn

${ }^{*}$ Contributed equally to this work.

Received October 13, 2021 / Accepted January 17, 2022

\begin{abstract}
Circular RNA is related to the tumorigenesis of various cancers. Circular RNA hsa_circ_0020123 (circ_0020123) has been uncovered to promote non-small cell lung cancer (NSCLC) progression. However, the regulatory mechanism of circ_0020123 in NSCLC is unclear. The quantitative real-time polymerase chain reaction was employed to detect the levels of circ_0020123, microRNA (miR)-193a-3p, and IRF4 interferon regulatory factor 4 (IRF4) in NSCLC tissues and cells. Loss-of-function experiments were performed to analyze the impacts of circ_0020123 silencing on NSCLC cell malignancy, autophagy, and glycolysis. Protein levels were detected using western blotting. The regulatory mechanism of circ_0020123 was analyzed by bioinformatics analysis and validated by the dual-luciferase reporter, RNA immunoprecipitation assay, and RNA pull-down assay. Xenograft assay was performed to verify the biological function of circ_0020123. We observed an overt elevation in circ_0020123 expression in NSCLC samples and cells, and NSCLC patients with high circ_0020123 expression had a poor prognosis. Circ_0020123 knockdown constrained xenograft tumor growth in vivo and curbed cell proliferation, migration, and glycolysis, and accelerated cell apoptosis and autophagy in NSCLC cells in vitro. Circ_0020123 could absorb miR-193a-3p to regulate IRF4 expression. miR-193a-3p silencing overturned circ_0020123 knockdownmediated impacts on NSCLC cell malignancy, autophagy, and glycolysis. And IRF4 overexpression reversed miR-193a-3p mimic-mediated effects on NSCLC cell malignancy, autophagy, and glycolysis. Circ_0020123 promoted glycolysis and tumor growth by upregulating IRF4 through sequestering miR-193a-3p in NSCLC, offering a novel mechanism by which circ_0020123 is responsible for the malignancy, autophagy, and glycolysis of NSCLC cells.
\end{abstract}

Key words: NSCLC; circ_0020123; miR-193a-3p; IRF4

Non-small cell lung cancer (NSCLC), which includes larger cell carcinoma, squamous cell carcinoma, and adenocarcinoma, is the leading cause of cancer-related deaths worldwide [1-3]. Also, based on surgery, drug therapy, and radiotherapy, the 5-year survival rate of stage I NSCLC has increased to $67 \%$ [4]. However, a large number of NSCLC patients are diagnosed at an advanced stage, so they cannot undergo radical surgery and have a poor five-year survival rate [5]. Therefore, it is important to explore the mechanism of NSCLC progression to provide direction for the development of new NSCLC treatment strategies.

Circular RNAs (circRNAs), novel promising biomarker candidates, are characterized by a covalently closed loop structure $[6,7]$. Compared with linear RNAs, circRNAs are resis- tant to RNase R and have a longer half-life [8]. Also, circRNAs can function as microRNA (miR) sponges, regulators of the parental gene, and regulators of splicing and transcription [9]. Mounting studies have unmasked that circRNAs mediate the progression of human diseases through binding to miRs via miR response elements, thus reducing the activity of miRs [10, 11]. For instance, circRNA 0001829 facilitated gastric cancer tumorigenesis by adsorbing miR-155-5p and increasing SMAD2 expression [12]. CircRNA UCK2 reduced oxygen-glucose deprivation-induced apoptosis by regulation of the miR-125b-5p/GDF11 pathway in cerebral ischemiareperfusion injury [13]. CircRNA hsa_circ_0020123 (circ_0020123), located at chr10:119042605-119049859, is derived from the PDZ domain containing 8 (PDZD8) gene 
(http://www.circbank.cn/search.html? selectValue=hsa circ_0020123). Circ_0020123 has been reported to facilitate the growth of NSCLC [14]. Nevertheless, the molecular mechanism that circ_0020123 promotes NSCLC progression is still unclear.

miRs control various developmental and cellular processes in eukaryotic organisms [15]. Functional studies have proved that $\mathrm{miR}$ dysregulation is related to the occurrence of many cancers $[16,17]$. miR-193a-3p plays a tumor-inhibiting/promoting role in many cancers [18-20]. Furthermore, miR-193a-3p has an anti-tumor activity in NSCLC $[21,22]$. However, the molecular mechanism of miR-193a-3p dysregulation in NSCLC remains unclear.

One of the hallmarks of cancer is changes in energy metabolism. This metabolism of cancer preferentially depends on glycolysis (the process of converting glucose into pyruvate and then producing lactic acid) [23]. The metabolic intermediates of glycolysis also promote the biosynthesis of macromolecules, which further facilitate the growth of cancer cells [24].

The study aimed to survey the regulatory mechanism by which circ_0020123 regulated glycolysis and NSCLC progression. Our results exhibited that circ_0020123 promoted glycolysis and NSCLC progression by sponging miR-193a-3p and upregulating interferon regulatory factor 4 (IRF4).

\section{Patients and methods}

Clinical specimens and ethics statement. All humanrelated procedures were carried out in accordance with the Declaration of Helsinki, and the study was approved by the Ethics Committee of the Sixth Affiliated Hospital of Sun Yat-sen University with number L2020ZSLYEC-024. 30 pairs of NSCLC tissues and adjacent normal tissues were obtained from the Sixth Affiliated Hospital of Sun Yat-sen University. All recruited patients with NSCLC had signed informed consents and did not receive chemotherapy or radiotherapy before surgery.

Table 1. Primer sequences used for $\mathrm{qRT}$-PCR.

\begin{tabular}{|c|c|}
\hline Genes & Primer sequences (5'-3') \\
\hline \multirow[t]{2}{*}{ circ_0020123 } & Forward: 5'-GTATGCACTCTGGCCTGCTT-3' \\
\hline & Reverse: 5'-ACCCATCAGTTGACTGGACA-3' \\
\hline \multirow[t]{2}{*}{ PDZD8 } & Forward: 5'-CTCCAAACTCGCCTGCTGCAAT-3' \\
\hline & R: 5'-CATAGTACACCAGGACTCGGTC-3' \\
\hline \multirow[t]{2}{*}{ IRF4 } & Forward: 5'-GAACGAGGAGAAGAGCATCTTCC-3' \\
\hline & Reverse: 5'-CGATGCCTTCTCGGAACTTTCC-3' \\
\hline \multirow[t]{2}{*}{$\beta$-actin } & Forward:5'-CTCGCCTTTGCCGATCC-3' \\
\hline & Reverse: 5'-TCTCCATGTCGTCCCAGTTG-3’ \\
\hline \multirow[t]{2}{*}{ miR-193a-3p } & Forward: 5'-CGCGAACTGGCCTACAAAGT-3' \\
\hline & Reverse: 5'AGTGCAGGGTCCGAGGTATT-3' \\
\hline \multirow[t]{2}{*}{ U6 } & Forward: 5'-CTCGCTTCGGCAGCACA-3' \\
\hline & Reverse: 5'-ACGCTTCACGAATTTGCGTGTC-3' \\
\hline
\end{tabular}

Cell culture. Human normal lung epithelial cells (BEAS2B) (COBIOER, Nanjing, China) and NSCLC cells (A549, H1299, and LTEP-s) (COBIOER) were maintained in a humidified air at $37^{\circ} \mathrm{C}$ with $5 \% \mathrm{CO}_{2}$. BEAS-2B cells were cultured in Lonza BEGM (Bronchial Epithelial Growth Medium) (Walkersville, MD, USA). A549, H1299, and LTEP-s cells were cultured in RPMI (Roswell Park Memorial Institute)-1640 medium (Sigma, St. Louis, MO, USA) supplemented with 10\% FBS (fetal bovine serum) (Thermo Fisher, Waltham, MA, USA) and $1 \%$ penicillin/streptomycin (Sigma).

Oligonucleotides and plasmids. Small interference (si) RNA against circ_0020123 (si-circ_0020123), short hairpin (sh) RNA against circ_0020123 (sh-circ_0020123), and their matching negative controls (NCs) (si-NC and sh-NC), miR-193a-3p inhibitor (anti-miR-193a-3p), miR-193a-3p mimic (miR-193a-3p), and their corresponding NCs (anti-miR-NC and miR-NC) were synthesized by AoKe Biotech (Beijing, China). For the generation of pCD5-ciRcirc_0020123 (circ_0020123) and pcDNA-IRF4 (IRF4) plasmids, the full-length sequences of circ_0020123 and IRF4 were synthesized and inserted into pCD5-ciR (Geneseed, Guangzhou, China) or pcDNA (Thermo Fisher) vectors, respectively. Transfection of NSCLC cells was performed with the Lipofectamine 3000 reagent (Thermo Fisher).

Quantitative real-time polymerase chain reaction (qRT-PCR). Total RNA was isolated using the RNeasy Mini Kit (QIAGEN, Hilden, Germany). For RNA digestion, total

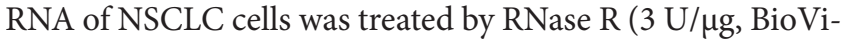
sion, Milpitas, CA, USA), and DEPC (diethylpyrocarbonate)treated water (Thermo Fisher) was used as a mock. For transcriptional inhibition, the culture medium of NSCLC cells was supplemented with actinomycin D, and DMSO (dimethyl sulfoxide) was utilized as a control. For complementary DNA, total RNA (500 ng) was reversely transcribed using the Prime-Script RT reagent kit (TaKaRa, Dalian, China) or TaqMan miRNA Reverse Transcription Kit (Applied Biosystems, Foster City, CA, USA). qRT-PCR was performed with the SYBR Green PCR Master Mix (Applied Biosystems). $\beta$-actin and U6 were used as internal references. Relative expression was calculated by the $2^{-\Delta \Delta \mathrm{Ct}}$ method. All primer sequences were listed in Table 1. Each experiment was performed in triplicate.

Cell proliferation analysis. The proliferation of NSCLC cells was evaluated by MTT (3-(4,5-dimethylthiazol-2-yl)2,5-diphenyltetrazolium bromide) and colony formation assays. For the MTT assay, NSCLC cells were transfected with oligonucleotides and/or plasmids and then cultured in 96 -well plates $\left(1 \times 10^{3} /\right.$ well $)$ for different times. Then, the MTT reagent $(20 \mu \mathrm{l})$ (Beyotime, Jiangsu, China) was added. After discarding the supernatant, the precipitated crystals were dissolved with DMSO (150 $\mu$ l, Beyotime), followed by measuring the OD (optical density) value (490 nm) using a microplate detection system (Molecular Devices, San Jose, 
CA, USA). For the colony formation assay, the transfected NSCLC cells $\left(5 \times 10^{2} /\right.$ well $)$ were cultured in 6 -well plates for 10 days. Then, these colonies were fixed by $10 \%$ formaldehyde (Sigma), followed by staining with $0.5 \%$ crystal violet (Sigma). The colonies were counted using a microscope (Nikon Instruments, Melville, NY, USA). Each experiment was performed in triplicate.

Flow cytometry assay. Flow cytometry assay was performed using Cytoflex Flow Cytometry (Beckman Coulter, Brea, CA, USA). In brief, the transfected NSCLC cells were collected and stained using the Annexin V-FITC (Fluorescein Isothiocyanate)/Propidium Iodide (PI) Apoptosis Detection Kit (Thermo Fisher) following the manufacturer's instructions. The percentage of apoptotic cells was analyzed using the FlowJo software (Tree Star, San Carlos, CA, USA).

Western blotting. The RIPA buffer containing protease and phosphatase inhibitors (Thermo Fisher) was utilized to extract total protein. Protein samples $(30 \mu \mathrm{g})$ were run on sodium dodecyl sulfate-polyacrylamide gel electrophoresis and then transferred to the polyvinylidene fluoride (PVDF) membrane (Bio-Rad, Hercules, CA, USA), followed by sealing in $5 \%$ non-fat milk. Then, the membranes were incubated with primary antibodies against p62 (\#sc-28359, 1:500), Beclin1 (\#sc-48381, 1:500), TRF4 (\#sc-81637, 1:500), and $\beta$-actin (\#sc-58673, 1:500), respectively. All primary antibodies were bought from Santa Cruz (Santa Cruz, CA, USA). Mouse-IgGk BP-HRP (horseradish peroxidase) (Santa Cruz) was used as the secondary antibody. The blots were developed using the Western Blotting Luminol Reagent (Santa Cruz).

Transwell migration assay. In brief, $200 \mu \mathrm{l}$ of serum-free cell culture medium containing transfected NSCLC cells $\left(1 \times 10^{5} /\right.$ chamber $)$ was added to the upper Transwell chambers (\#3422, Costar, Cambridge, MA, USA). $600 \mu$ of cell culture medium containing $10 \%$ FBS (Thermo Fisher) was added to the lower Transwell chambers (\#3422, Costar). 24 h later, the migrating cells were stained with $0.5 \%$ crystal violet (Sigma) and then calculated under a microscope (Nikon Instruments) $(\times 100$ amplification $)$ in 5 random regions.

Metabolic analysis. The levels of glucose consumption, lactate production, and ATP (adenosine triphosphate) release were assessed using the Glucose Uptake Colorimetric Assay kit (BioVision), Lactate Assay Kit II (BioVision), and ATP Colorimetric Assay kit (BioVision) according to the manufacturer's instructions.

Extracellular acidification rate (ECAR) was evaluated using the Seahorse XF Glycolysis Stress Test Kit (Seahorse Bioscience, Billerica, MA, USA) on the Seahorse XFe 96 Extracellular Flux Analyzer (Seahorse Bioscience). For the ECAR detection, glucose, oligomycin, and 2-DG (2-deoxyglucose) were added to Seahorse XF 96 microtiter plates in sequence. ECAR was reported in $\mathrm{mpH} / \mathrm{min}$.

Dual-luciferase reporter assay. To validate these predictions, the sequences of circ_0020123-MUT (mutant),
circ_0020123-WT (wild type), IRF4 3'UTR (untranslated region)-MUT, and IRF4 3'UTR-WT were synthesized by AoKe Biotech and then inserted into the pMIR-REPORT reporter (Applied Biosystems), respectively. The luciferase activity in NSCLC cells co-transfected with miR-193a-3p mimic or miR-NC and a luciferase reporter was evaluated using a dual-luciferase reporter assay kit (BioVision).

RIP (RNA immunoprecipitation) assay. In brief, the lysates of NSCLC cells were incubated with immunoprecipitation buffer containing magnetic beads conjugated to IgG antibody (ab172730, Abcam, Cambridge, MA, USA) or Ago2 antibody (ab186733, Abcam). The enrichment of circ_0020123 or TRF4 and miR-193a-3p was assessed by qRT-PCR. RIP assay was carried out with the Magna RIP kit (Millipore, Bedford, MA, USA) based on the manufacturer's instructions.

RNA pull-down assay. Transfection of NSCLC cells Bio-miR-193a-3p or Bio-NC probe was performed. The lysates of NSCLC cells were incubated with M-280 streptavidin magnetic beads (Thermo Fisher), which were pre-blocked using yeast tRNA (Thermo Fisher). The enrichment of circ_0020123 in precipitates was detected using qRT-PCR.

Xenograft assay. To generate A549 cells with stable knockdown of circ_0020123, the sequence of sh-circ_0020123 was inserted into the pLKO.1 vector (Thermo Fisher), followed by transfecting into HEK293T cells (COBIOER) together with lentiviral packaging plasmids using the Lipofectamine 3000 reagent (Thermo Fisher). The sh-NC was used as a control. Subsequently, A549 cells were infected with lentiviral particles produced by HEK293T cells and then selected with puromycin $(2 \mu \mathrm{g} / \mathrm{ml}$, Sigma).

The animal experiments were approved by the Animal Ethics Committee of The Sixth Affiliated Hospital of Sun Yat-sen University. For the xenograft assay, A549 cells with sh-circ_0020123 or sh-NC were subcutaneously injected into the right flank of BALB/c nude mice (4-6 weeks old, 15-20 g), which were purchased from Vital River Laboratory (Beijing, China) and divided into 2 groups randomly $(n=5$ mice/group). After injection for 4 weeks, the tumor tissues of the euthanized mice were stripped for tumor weight assessment and gene expression analysis. Tumor volume was measured once a week and calculated based on the following equation: Volume $=\left(\right.$ length $\times$ width $\left.^{2}\right) / 2$.

Statistical analysis. Data from three independent experiments were presented as mean \pm standard deviation. GraphPad Prism 7 software (GraphPad Inc., La Jolla, CA, USA) was utilized for statistical analyses. Unpaired or paired Student's t-test was used to analyze the difference between the 2 groups. Analysis of variance was used to evaluate analyze the differences in three or more groups. Overall survival was assessed by Kaplan-Meier and log-rank test. Pearson's correlation analysis was used to analyze the correlation among circ_0020123, miR-193a-3p, and TRF4. All statistical tests were considered significant when $\mathrm{p}<0.05$. 


\section{Results}

Circ_0020123 was highly expressed in NSCLC. A previous microarray profile displayed that hsa_circ_0020123 was overexpressed in NSCLC tissues [25]. To survey the change in circ_0020123 expression in NSCLC, we detected the expression tendency of circ_0020123 in NSCLC. The results exhibited that circ_0020123 expression was observably higher in NSCLC tissues than that in adjacent normal tissues (Figure 1A). Analogously, there was a marked increase in circ_0020123 expression in NSCLC cells (A549, H1299, and LTEP-s) compared to the BEAS-2B cells (Figure 1B). Moreover, the overall survival of NSCLC patients with high circ_0020123 expression was worse (Figure 1C). We also analyzed the stability of circ_0020123. As exhibited in Figures 1D and 1E, circ_0020123 was more stable than linear PDZD8. Also, the half-life of circ_0020123 was overtly longer than linear PDZD8 (Figures 1F, 1G). Together, these results indicated that circ_0020123 might be related to the progression of NSCLC.

Circ_0020123 repressed autophagy, facilitated malignant behaviors, and elevated glycolysis of NSCLC cells. To investigate the effects of circ_0020123 inhibition on malignant behaviors, glycolysis, and autophagy of NSCLC cells, we designed the siRNA targeting circ_0020123 to silence circ_0020123. The interference efficiency of si-circ_0020123 is presented in Figure 2A. Moreover, circ_0020123 silencing repressed the proliferation of A549 and H1299 cells in MTT and colony formation assays (Figures 2B-2E). Also, circ_0020123 knockdown accelerated cell apoptosis in A549 and H1299 cells in flow cytometry assay (Figures 2F, 2G). Furthermore, circ_0020123 knockdown reduced p62 protein levels and elevated Beclin1 protein levels in A549 and H1299 cells, implying that circ_0020123 inhibition facilitated NSCLC cell autophagy (Figures 2H, 2I). Transwell migration assay presented that the migration of A549 and H1299 cells was curbed after circ_0020123 knockdown (Figures 2J, 2K). In addition, circ_0020123 inhibition led to an overt decrease in glucose consumption, lactate production, and ATP release in A549 and H1299 cells (Figures 2L-2N). As expected, the ECAR of si-circ_0020123-transfected A549 and H1299 cells was overtly lower than the control cells (Figures 2O,2P). Collectively, circ_0020123 repressed autophagy, facilitated malignant behaviors, and elevated glycolysis of NSCLC cells.

Circ_0020123 served as a sponge for miR-193a-3p. Subsequently, we surveyed the regulatory mechanism of circ_0020123 in NSCLC. Online database starBase (http://starBase.sysu.edu.cn/) prediction exhibited that circ_0020123 possessed binding sites complementary to miR-193a-3p, suggesting that circ_0020123 might be a sponge for miR-193a-3p (Figure 3A). And the luciferase activity of the circ_0020123-WT reporter was repressed in miR-193a-3p-overexpressed cells, whereas the luciferase activity of the circ_0020123-MUT reporter did not



$E$

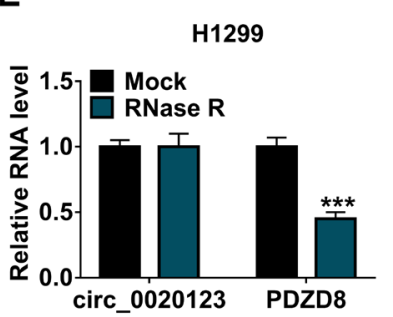

B



$\mathbf{F}$

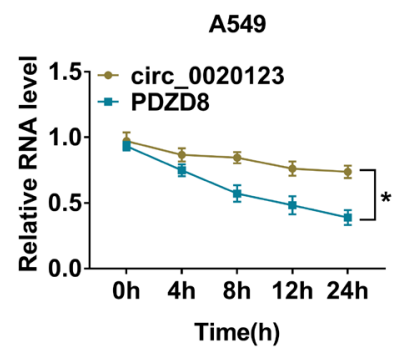

C
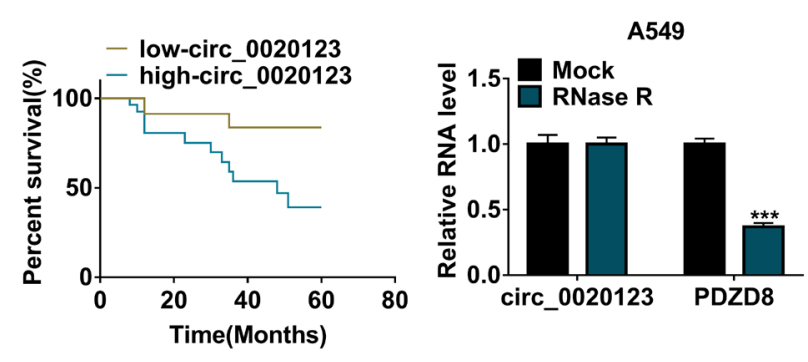

G



Figure 1. Circ_0020123 expression was elevated in NSCLC. A, B) qRT-PCR showed the expression trend of circ_0020123 in NSCLC tissues and cells. C) Kaplan-Meier survival plots analyzed the overall survival of NSCLC patients with high $(\geq \mathbf{m e d i a n}, \mathbf{n}=15)$ or low $(<\operatorname{median}$, $n=15)$ expression of circ_0020123. D, E) qRT-PCR revealed the levels of circ_0020123 and PDZD8 mRNA in total RNA (A549 and H1299 cells) under RNase R treatment. F, G) qRT-PCR detected the levels of circ_0020123 and PDZD8 mRNA in A549 and H1299 cells treated with actinomycin D. ${ }^{\star}$ p $<0.05$ and ${ }^{\star * *}$ p $<0.001$ 


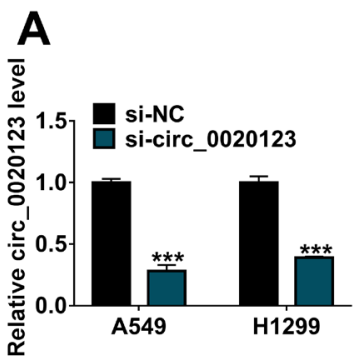

B



F



I

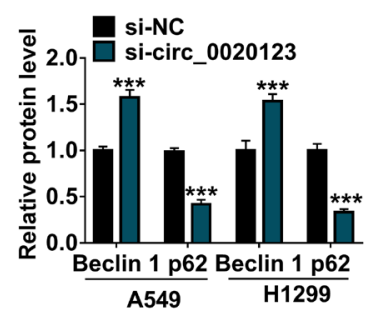

M
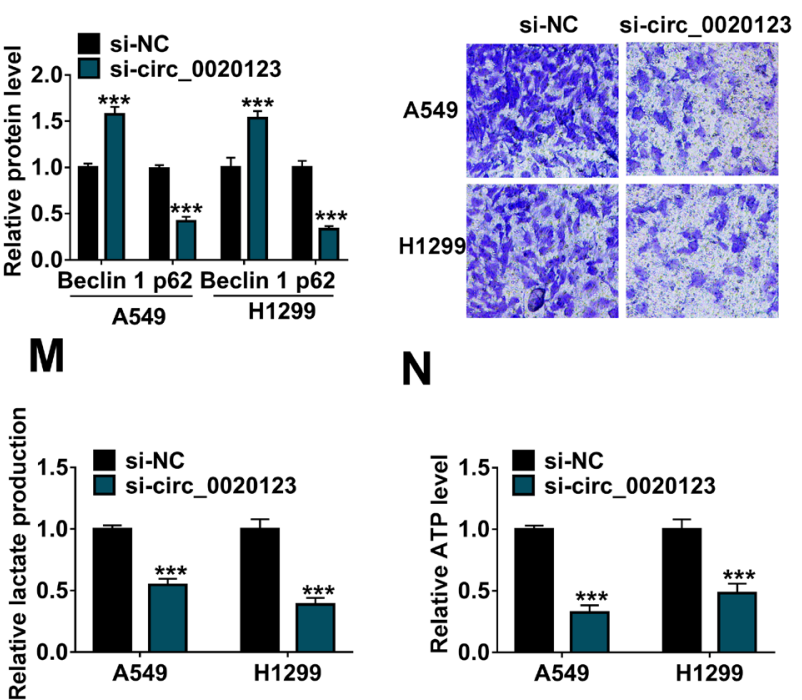

$\mathbf{N}$
C

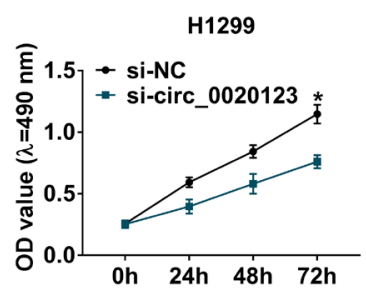

G
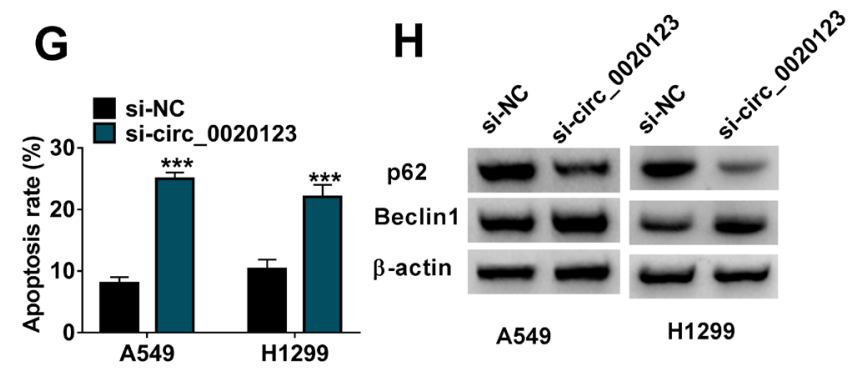

K



L

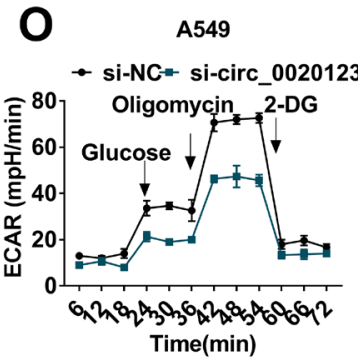

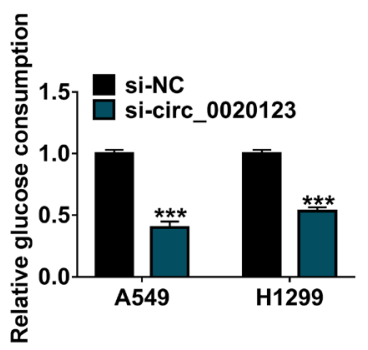

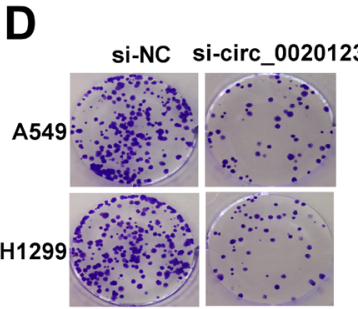

P

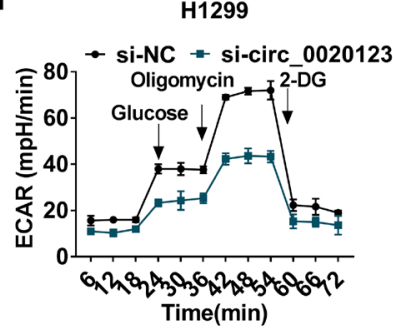

Figure 2. Circ_0020123 curbed autophagy and contributed to malignant behaviors and glycolysis of NSCLC cells. A) qRT-PCR verified the interference efficiency of si-circ_0020123 in A549 and H1299 cells. B-G) Influence of circ_0020123 inhibition on proliferation and apoptosis of A549 and H1299 cells was analyzed by MTT, colony formation, and flow cytometry assays. H, I) Western blotting exhibited protein levels of p62 and Beclin1 in A549 and H1299 cells transfected with si-circ_0020123. J, K) Impact of circ_0020123 silencing on the migration of A549 and H1299 cells was determined by Transwell assay. L-N) The levels of glucose consumption, lactate production, and ATP release in si-circ_0020123-transfected A549 and H1299 cells were evaluated using matching kits. O, P) Assessment of the ECAR of si-circ_0020123-transfected A549 and H1299 cells by using the Seahorse XF96 Extracellular Flux Analyzer, and si-NC was utilized as a control. ${ }^{\star} \mathrm{p}<0.05$ and ${ }^{\star * \star} \mathrm{p}<0.001$

change (Figure 3B). RIP exhibited that circ_0020123 and miR-193a-3p were co-enriched in the Ago2 group but not in the IgG group (Figures 3C, 3D). RNA pull-down assay exhibited that the enrichment of circ_0020123 was higher in RNA complexes pulled down by the Bio-miR-193a-3p probe than the Bio-NC probe (Supplementary Figure S1). Moreover, circ_0020123 was markedly downregulated in NSCLC tissues and cells (Figures 3E, 3F), and the expres- sion of circ_0020123 and miR-193a-3p in NSCLC tissues had a negative correlation (Figure $3 \mathrm{G}$ ). Also, the transfection efficiency of the circ_0020123 overexpression plasmid was validated in Figure 3H. circ_0020123 silencing led to an elevation in miR-193a-3p expression in A549 and H1299 cells, but circ_0020123 overexpression exerted a reverse effect (Figures 3I, 3J). These results manifested that circ_0020123 acted as a sponge for miR-193a-3p in NSCLC cells. 
Circ_0020123 adsorbed miR-193a-3p to regulate NSCLC cell malignant behaviors, autophagy, and glycolysis. We further explored whether circ_0020123 regulated malignant behaviors, autophagy, and glycolysis of NSCLC cells by miR-193a-3p. The results exhibited that transfection of miR-193a-3p inhibitor weakened the elevated expression of miR-193a-3p in NSCLC cells mediated by circ_0020123 knockdown (Figure 4A). Moreover, miR-193a-3p inhibitor partly counteracted circ_0020123 knockdown-mediated suppression on A549 and H1299 cell proliferation (Figures 4B-4D). Also, circ_0020123 knockdown-mediated effects on A549 and H1299 cell apoptosis and autophagy were impaired after miR-193a-3p silencing (Figures 4E-4I). Furthermore, the inhibitory influence of circ_0020123 knockdown on NSCLC cell migration was offset by miR-193a-3p silencing (Figure 4J). In addition, miR-193a-3p knockdown alleviated the decrease in glucose consumption, lactate production, ATP release, and ECAR in A549 and H1299 cells caused by circ_0020123 silencing (Figures 4K-4O). In summary, circ_0020123 regulated malignant behaviors, autophagy, and glycolysis of NSCLC cells by sponging miR-193a-3p.

IRF4 acted as a downstream target of miR-193a-3p. We further sought the downstream targets of miR-193a-3p
A

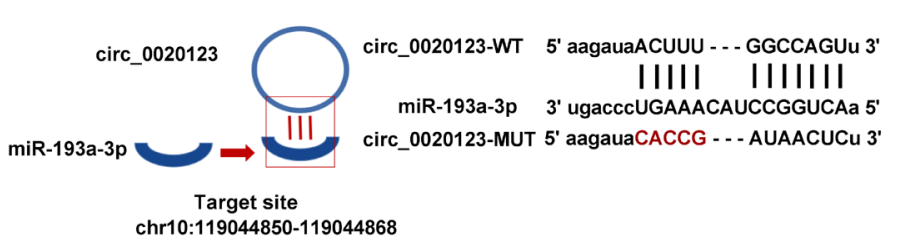

chr10:119044850-119044868

C

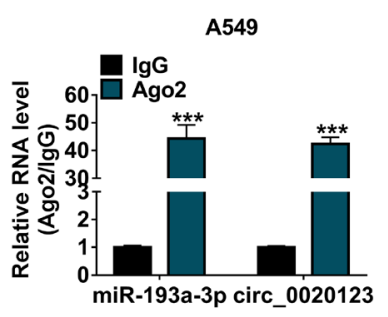

G



B
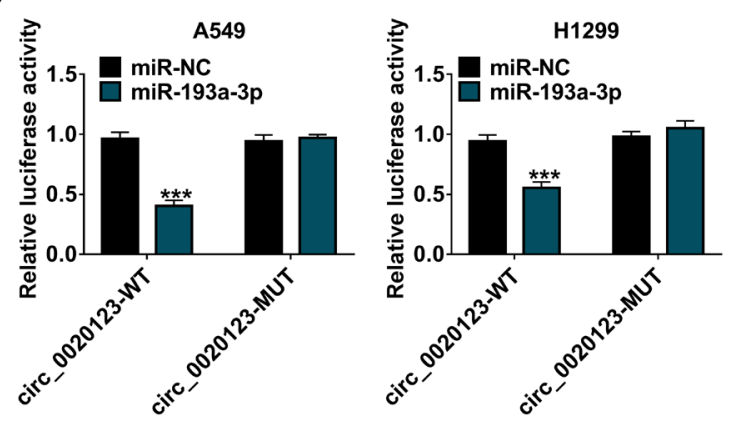

$\mathbf{F}$

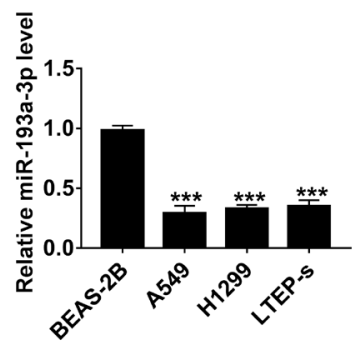

J
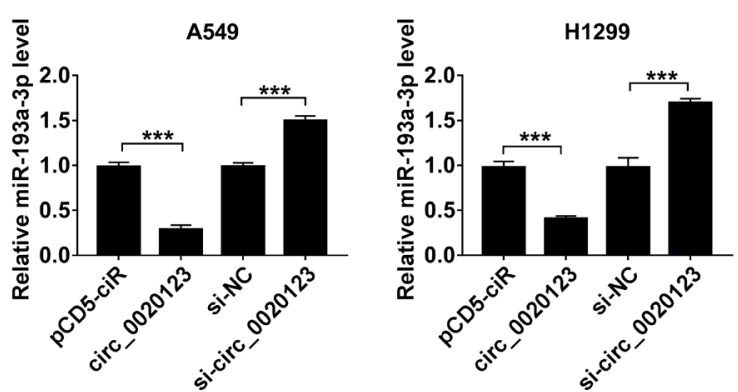

Figure 3. Circ_0020123 acted as a sponge for miR-193a-3p. A) The binding sites of circ_0020123 on miR-193a-3p. B) The binding sites between circ_0020123 and miR-193a-3p were verified by a dual-luciferase reporter assay. C, D) After RIP experiments, the enrichment of circ_0020123 and miR-193a-3p was analyzed by qRT-PCR. E, F) Expression of miR-193a-3p in NSCLC tissues and cells was assessed by qRT-PCR. G) The correlation between circ_0020123 and miR-193a-3p in NSCLC tissues was analyzed by Pearson's correlation analysis. (H) The overexpression efficiency of circ_0020123 was validated by qRT-PCR. I, J) Influence of circ_0020123 overexpression and inhibition on the expression of miR-193a-3p in A549 and H1299 cells were analyzed by qRT-PCR. ${ }^{* * *}$ p $<0.001$ 

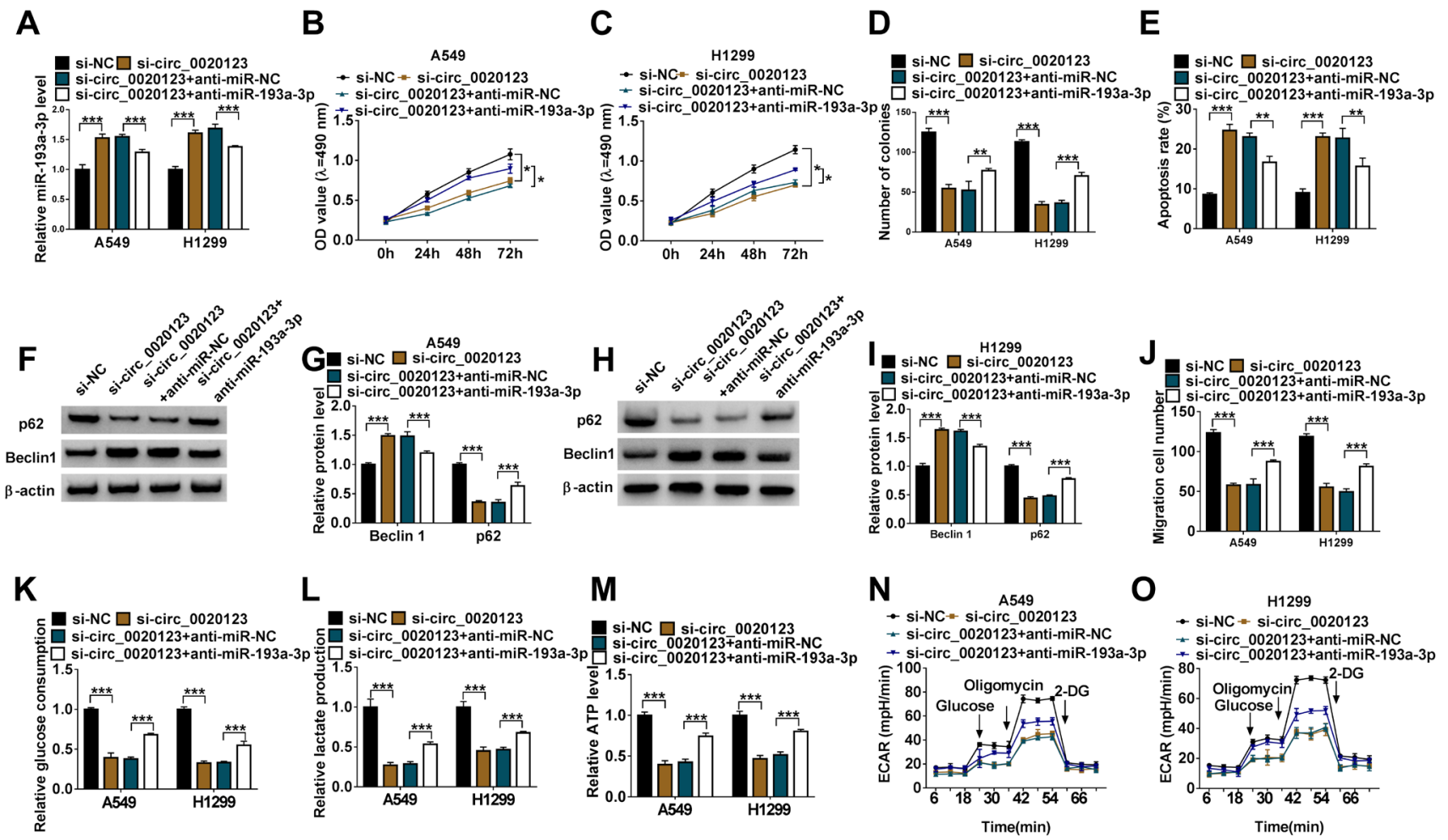

Figure 4. Circ_0020123 regulated NSCLC cell malignant behaviors, autophagy, and glycolysis through sponging miR-193a-3p. A-O) A549 and H1299 cells were transfected with si-NC, si-circ_0020123, si-circ_0020123+anti-miR-NC, or si-circ_0020123+anti-miR-193a-3p. A) Expression levels of miR193a-3p in A549 and H1299 cells were analyzed by qRT-PCR. B-E) The proliferation and apoptosis of A549 and H1299 cells were detected by MTT, colony formation, and flow cytometry assays. F-I) Protein levels of p62 and Beclin1 in A549 and H1299 cells were measured by western blotting. J) Transwell assay was utilized to assess the migration of A549 and H1299 cells. K-M) Measurement of glucose consumption, lactate production, and ATP release in A549 and H1299 cells using corresponding kits. N, O) Analysis of the ECAR in A549 and H1299 cells by using the Seahorse XF96 Extracellular Flux Analyzer. ${ }^{*} \mathrm{p}<0.05,{ }^{* *} \mathrm{p}<0.01$, and ${ }^{* * *} \mathrm{p}<0.001$

by online bioinformatics database starBase. As presented in Figure 5A, IRF4 might be a downstream target of miR-193a-3p. Moreover, miR-193a-3p mimic inhibited the luciferase activity of the IRF4 3'UTR-WT reporter but not the IRF4 3'UTR-MUT reporter (Figures 5B, 5C). Furthermore, the abundance of IRF4 and miR-193a-3p was higher in the Ago2 group (Figures 5D, 5E). And the expression of IRF4 mRNA was upregulated in NSCLC tissues, and it had a negative correlation with miR-193a-3p (Figures $5 \mathrm{~F}$ and $5 \mathrm{G})$. Congruously, IRF4 protein levels were upregulated in NSCLC cells and tissues (Figures 5H, 5I). In addition, the transfection efficiencies of miR-193a-3p inhibitor and mimic were verified and shown in Figure 5J. Also, miR-193a-3p overexpression repressed IRF4 protein levels, whereas miR-193a-3p silencing had an opposing effect (Figures 5K, 5L). In summary, these results indicated that miR-193a-3p directly targeted IRF4 in NSCLC cells.

miR-193a-3p targeted IRF4 to regulate NSCLC cell malignant behaviors, autophagy, and glycolysis. Knowing that IRF4 was a target of miR-193a-3p, we further explored whether IRF4 participated in malignant behaviors, autophagy, and glycolysis of NSCLC cells mediated by miR-193a-3p. And the decreased protein levels of IRF4 in miR-193a-3p-overexpressed A549 and H1299 cells were impaired after the introduction of the IRF4 overexpression plasmid (Figure 6A). Also, miR-193a-3p mimic repressed cell proliferation, accelerated cell apoptosis, and elevated cell autophagy, but these impacts were reversed after the IRF4 overexpression (Figures 6B-6I). Furthermore, IRF4 overexpression weakened the repressive effect of miR-193a-3p overexpression on the migration of A549 and H1299 cells (Figure 6J). Additionally, IRF4 upregulation reversed the decrease in glucose consumption, lactate production, ATP release, and ECAR in A549 and H1299 cells prompted by miR-193a-3p overexpression (Figure 6K-6O). Together, miR-193a-3p regulated NSCLC cell malignant behaviors, autophagy, and glycolysis by targeting IRF4.

Circ_0020123 regulated IRF4 expression by sponging miR-193a-3p. We further analyzed the relationship among circ_0020123, miR-193a-3p, and IRF4 in NSCLC. Pearson's correlation analysis exhibited that the expression of IRF4 mRNA was positively correlated with circ_0020123 in NSCLC tissues (Figure 7A). Moreover, circ_0020123 silencing reduced IRF4 protein levels, but this decrease 
A

IRF4 3' UTR-WT 5' cuUGGCCUUUCCUGGAAGCCAGUu 3' $\begin{array}{cc}\text { miR-193a-3p } & \text { 3' ugACCCUGAA - -ACAUCCGGUCAa 5' } \\ \text { IRF4 3' UTR-MUt } & \text { 5' cuCAACCUGGCCCAGCAAUUCACu 3' }\end{array}$
B

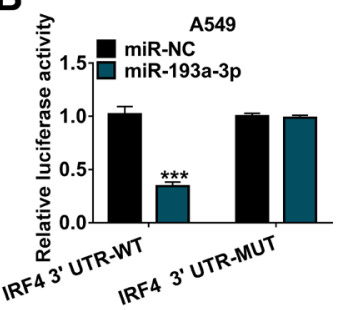

$F$


C



G

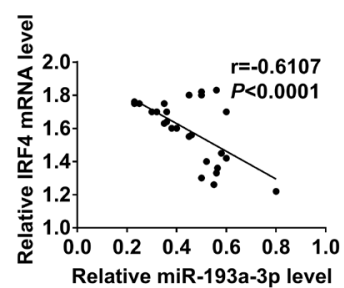

K

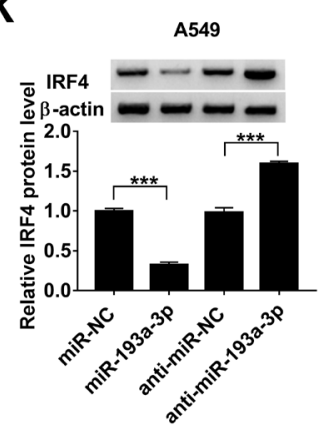



H

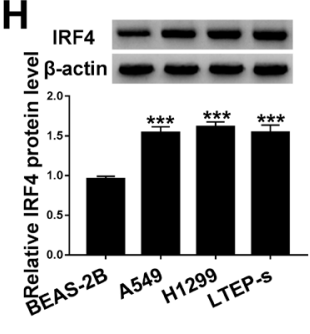

$\mathbf{L}$

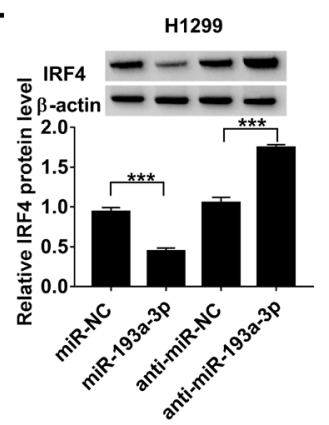

Figure 5. miR-193a-3p directly targeted IRF4 in NSCLC cells. A) The miR-193a-3p binding sites in IRF4 were predicted by the online bioinformatics database starBase. B, C) The binding sites between miR-193a-3p and IRF4 were confirmed by a dual-luciferase reporter assay. D, E) The abundance of miR-193a-3p and IRF4 in RISC (RNA-induced silencing complex) of the Ago2 group was analyzed by qRT-PCR. F) Expression level of IRF4 mRNA in NSCLC tissues was detected by qRT-PCR. G) Pearson's correlation analysis presented the correlation between IRF4 mRNA and miR-193a-3p in NSCLC tissues. H, I) Protein levels of IRF4 in NSCLC cells and tissues were analyzed by western blotting. J) Expression level of miR-193a-3p in A549 and H1299 cells transfected with miR-193a-3p or anti-miR-193a-3p was assessed by qRT-PCR. K, L) Effects of miR-193a-3p overexpression and knockdown on the protein level of IRF4 in A549 and H1299 cells were evaluated by western blotting. ${ }^{* * *} \mathrm{p}<0.001$

was overturned after anti-miR-193a-3p co-transfection (Figures 7B, 7C). In summary, these results indicated that circ_0020123 regulated IRF4 expression by sponging miR-193a-3p in NSCLC cells.

Circ_0020123 knockdown decreased xenograft tumor growth in vivo. To verify the function of circ_0020123 in NSCLC, we constructed xenograft models by injecting A549 cells with a stable knockdown of circ_0020123. The results exhibited that xenograft tumors in the sh-circ_0020123 group had a smaller size (volume) and lighter weight compared to the sh-NC group (Figures 8A-8C). Also, the levels of circ_0020123 and IRF4 protein were downregulated in xenograft tumors in the sh-circ_0020123 group compared with the control group, whereas miR-193a-3p expression had an opposite result (Figures 8D-8F). Collectively, these results indicated that circ_0020123 inhibition reduced NSCLC growth in vivo.

\section{Discussion}

In this report, we uncovered that circ_0020123 served as a decoy for miR-193a-3p, and circ_0020123 elevated IRF4 expression by sponging miR-193a-3p, resulting in repressing autophagy, accelerating malignancy, and elevating glycolysis in NSCLC cells.

Autophagy plays an important role in removing unnecessary organelles and misfolded proteins in lysosomes. Moreover, autophagy exerts a vital role in maintaining cell differentiation, remodeling, and homeostasis [26]. p62, also termed SQSTM1, is a ubiquitin-binding scaffold protein that can be accumulated when autophagy is inhibited [27]. Beclin-1, a key regulator of autophagy, plays an important role in crosstalk with the apoptotic pathway [28]. At present, the paradoxical role of autophagy in suppressing and promoting tumors has attracted widespread attention [29, 
A

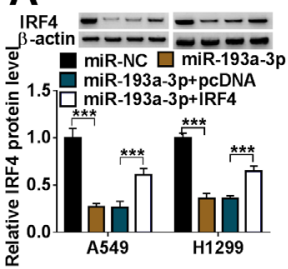

F



K

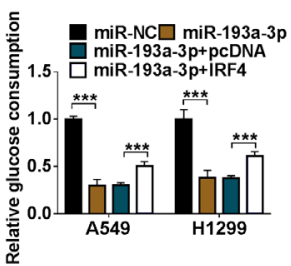

B
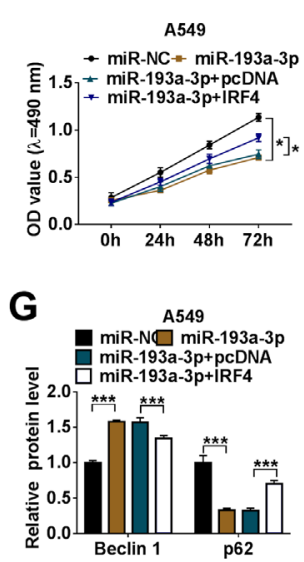

L

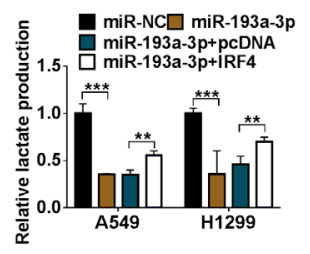

C
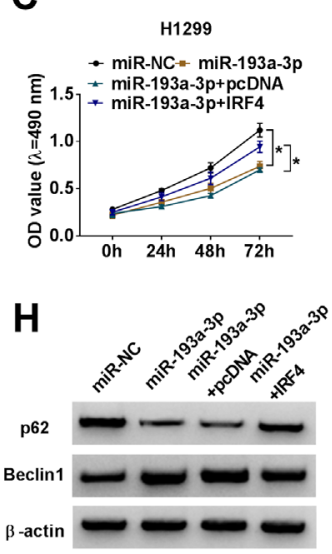

M

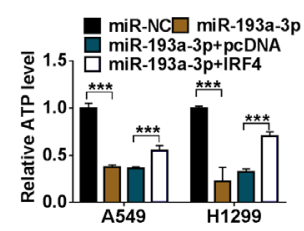

D
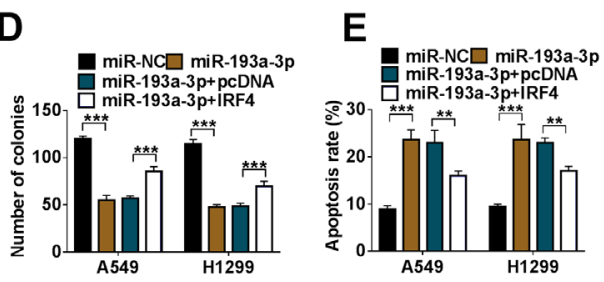

I

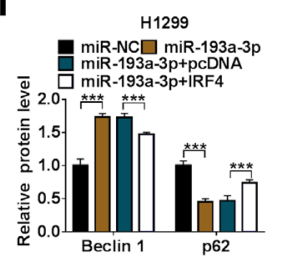

$\mathbf{N}$

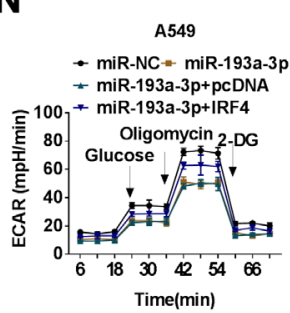



$\mathrm{O}$

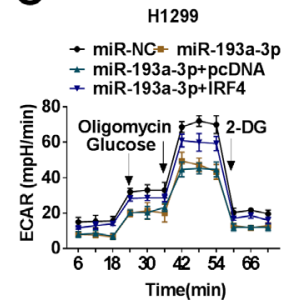

Figure 6. miR-193a-3p regulated NSCLC cell malignant behaviors, autophagy, and glycolysis by targeting IRF4. A-O) A549 and H1299 cells were transfected with miR-NC, miR-193a-3p, miR-193a-3p+pcDNA, or miR-193a-3p+IRF4. A) Analysis of the level of IRF4 protein in A549 and H1299 cells by western blotting. B-E) Assessment of proliferation and apoptosis of A549 and H1299 cells by MTT, colony formation, and flow cytometry assays. F-I) Detection of protein levels of p62 and Beclin1 in A549 and H1299 cells by western blotting. J) Transwell assay was performed to assess the migration of A549 and H1299 cells. K-M) The levels of glucose consumption, lactate production, ATP release in A549 and H1299 cells were determined by using corresponding kits. N, O) The ECAR of A549 and H1299 cells was analyzed using the Seahorse XF96 Extracellular Flux Analyzer. ${ }^{\star} p<0.05,{ }^{* *} \mathbf{p}<0.01$, and ${ }^{* * *} \mathbf{p}<0.001$
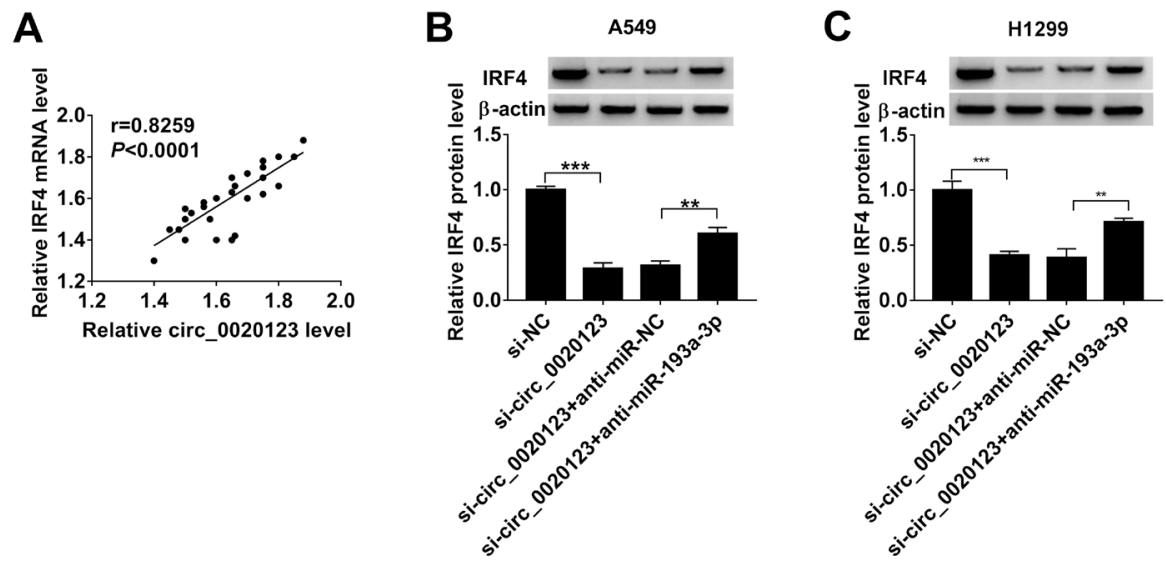

Figure 7. Circ_0020123 sponged miR-193a-3p to regulate IRF4 expression. A) Pearson's correlation analysis exhibited the correlation between circ_0020123 and IRF4 in NSCLC tissues. B, C) Western blotting detected the level of IRF4 protein in A549 and H1299 cells transfected with si-NC, si-circ_0020123, si-circ_0020123+anti-miR-NC, or si-circ_0020123+anti-miR-193a-3p. ${ }^{\star *} p<0.01$ and ${ }^{* * *} p<0.001$

30]. A previous study revealed that circRNA circ_0085131 elevated cell autophagy and cisplatin resistance by regulating the miR-654-5p/ATG7 pathway in NSCLC [31]. In contrast, circRNA circHIPK3 downregulation repressed cell malignancy and induced cell autophagy by sponging miR124-3p and downregulating STAT3 in NSCLC [32]. Herein, NSCLC patients with high circ_0020123 expression had a poor prognosis, manifesting that circ_0020123 might be an underlying prognostic biomarker for NSCLC. Furthermore, circ_0020123 silencing accelerated NSCLC cell apoptosis, 

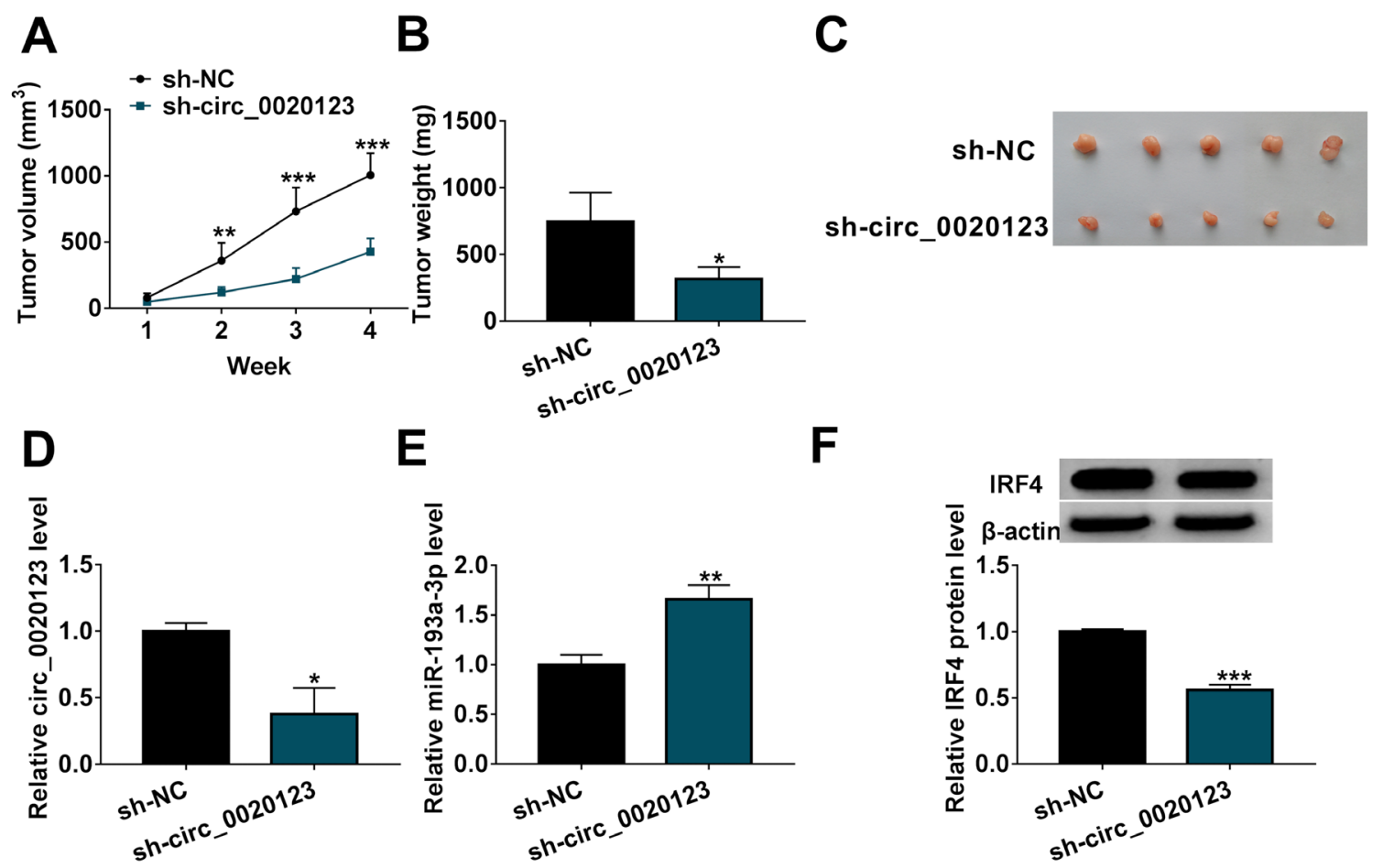

Figure 8. Knockdown of circ_0020123 decreased NSCLC growth in vivo. A) The volume of xenograft tumors in nude mice with sh-circ_0020123 or sh-NC. B) The weight of xenograft tumors in nude mice with sh-circ_0020123 or sh-NC. C) A picture containing all xenograft tumors in nude mice. D-F) The levels of circ_0020123, miR-193a-3p, and IRF4 protein in xenograft tumors in nude mice with sh-circ_0020123 or sh-NC were detected by qRT-PCR or western blotting. ${ }^{*} \mathrm{p}<0.05,{ }^{* *} \mathrm{p}<0.01$, and ${ }^{* * *} \mathrm{p}<0.001$

autophagy, and repressed NSCLC cell proliferation, migration, and glycolysis. In vivo experiments, circ_0020123 inhibition decreased the growth of xenograft tumors. Qu et al. uncovered that circ_0020123 sponged miR-144 to increase EZH2 and ZEB1 expression, resulting in accelerating NSCLC cell growth and metastasis [14]. Also, circ_0020123 could accelerate NSCLC progression by regulating miR-495/ HOXC9 [33] and miR-590-5p/THBS2 [34] pathways. In addition, circ_0020123 (circPDZD8) could promote gastric cancer cell migration and proliferation by indirectly upregulating CHD9 via sequestering miR-197-5p [35]. Therefore, we concluded that circ_0020123 repressed cell autophagy and facilitated cell glycolysis and malignancy in NSCLC.

Based on the ceRNA (competing endogenous RNA) hypothesis [3], circ_0020123 was validated as a miR-193a-3p sponge in the research. miR-193a-3p has been revealed to play an inhibitory influence on cell malignancy in diverse cancers, such as colorectal cancer [36] and prostate cancer [37]. Also, miR-193a-3p targeted PAK4 to block the p53/Slug/L1CAM pathway, leading to repressing tumor growth and metastasis in NSCLC [38]. In addition, miR-193a-3p/-5p curbed NSCLC cell metastasis by targeting the ERBB signaling [21]. Herein, miR-193a-3p was lowly expressed in NSCLC. Moreover, miR-193a-3p overturned circ_0020123 inhibition-mediated effects on NSCLC cell malignancy, glycolysis, and autophagy. These results manifested that circ_0020123 inhibited NSCLC cell autophagy and accelerated NSCLC cell glycolysis and malignancy through sponging miR-193a-3p.

IRF4, a member of the IRF family, plays a vital role in regulating the immune response [39]. IRF4 has been revealed to be a potential diagnostic and prognostic biomarker in various hematological malignancies [40]. IRF4-induced SOX2-OT accelerated cholangiocarcinoma cell metastasis and proliferation via activation of the PI3K/ AKT pathway by elevating IRF4 expression [41]. Also, IRF4 promoted cell proliferation by activating the NOTCHAKT pathway in NSCLC [42]. Herein, IRF4 was identified as a miR-193a-3p target. Furthermore, IRF4 overexpression overturned miR-193a-3p mimic-mediated impacts on NSCLC cell autophagy, malignancy, and glycolysis. Importantly, circ_0020123 adsorbed miR-193a-3p to increase IRF4 expression. Therefore, we inferred that circ_0020123 curbed cell autophagy, increased cell glycolysis, and promoted cell malignancy by regulating the miR-193a-3p/IRF4 axis in NSCLC.

In summary, circ_0020123 exerted an oncogenic role in NSCLC. Mechanically, circ_0020123 acted as a miR-193a-3p sponge and elevated IRF4 expression by adsorbing miR-193a-3p, resulting in repressing NSCLC cell autophagy and accelerating NSCLC cell malignancy and glycolysis. 
The research offered a new mechanism that circ_0020123 is responsible for the advancement of NSCLC.

Supplementary information is available in the online version of the paper.

\section{References}

[1] DESANTIS CE, MILLER KD, GODING SAUER A, JEMAL A, SIEGEL RL. Cancer statistics for African Americans, 2019. CA Cancer J Clin 2019; 69: 211-233. https://doi. org/10.3322/caac. 21555

[2] HERREROS-POMARES A, DE-MAYA-GIRONES JD, CALABUIG-FARIÑAS S, LUCAS R, MARTÍNEZ A et al. Lung tumorspheres reveal cancer stem cell-like properties and a score with prognostic impact in resected non-small-cell lung cancer. Cell Death Dis 2019; 10: 660. https://doi.org/10.1038/ s41419-019-1898-1

[3] WANG M, MAO C, OUYANG L, LIU Y, LAI W et al. Long noncoding RNA LINC00336 inhibits ferroptosis in lung cancer by functioning as a competing endogenous RNA. Cell Death Differ 2019; 26: 2329-2343. https://doi.org/10.1038/ s41418-019-0304-y

[4] DETTERBECK FC, BOFFA DJ, TANOUE LT. The new lung cancer staging system. Chest 2009; 136: 260-271. https://doi. org/10.1378/chest.08-0978

[5] REMON J, HENDRIKS LE, CABRERA C, REGUART N, BESSE B. Immunotherapy for oncogenic-driven advanced non-small cell lung cancers: Is the time ripe for a change? Cancer Treat Rev 2018; 71: 47-58. https://doi.org/10.1016/j. ctrv.2018.10.006

[6] VO JN, CIESLIK M, ZHANG Y, SHUKLA S, XIAO L et al. The Landscape of Circular RNA in Cancer. Cell 2019; 176. https://doi.org/10.1016/j.cell.2018.12.021

[7] ZHANG Y, LIANG W, ZHANG P, CHEN J, QIAN H et al. Circular RNAs: emerging cancer biomarkers and targets. J Exp Clin Cancer Res 2017; 36: 152. https://doi.org/10.1186/ s13046-017-0624-Z

[8] SALZMAN J. Circular RNA Expression: Its Potential Regulation and Function. Trends Genet 2016; 32: 309-316. https:// doi.org/10.1016/j.tig.2016.03.002

[9] QU S, YANG X, LI X, WANG J, GAO Y et al. Circular RNA: A new star of noncoding RNAs. Cancer Lett 2015; 365: 141148. https://doi.org/10.1016/j.canlet.2015.06.003

[10] QI X, ZHANG DH, WU N, XIAO JH, WANG X et al. ceRNA in cancer: possible functions and clinical implications. J Med Genet 2015; 52: 710-718. https://doi.org/10.1136/jmedgenet-2015-103334

[11] ABDOLLAHZADEH R, DARAEI A. Competing endogenous RNA (ceRNA) cross talk and language in ceRNA regulatory networks: A new look at hallmarks of breast cancer. J Cell Physiol 2019; 234: 10080-10100. https://doi. org/10.1002/jcp.27941

[12] NIU Q, DONG Z, LIANG M, LUO Y, LIN H et al. Circular RNA hsa_circ_0001829 promotes gastric cancer progression through miR-155-5p/SMAD2 axis. J Exp Clin Cancer Res 2020; 39: 280. https://doi.org/10.1186/s13046-020-01790-w
[13] CHEN W, WANG H, FENG J, CHEN L. Overexpression of circRNA circUCK2 Attenuates Cell Apoptosis in Cerebral Ischemia-Reperfusion Injury via miR-125b-5p/GDF11 Signaling. Mol Ther Nucleic Acids 2020; 22: 673-683. https:// doi.org/10.1016/j.omtn.2020.09.032

[14] QU D, YAN B, XIN R, MA T. A novel circular RNA hsa circ_0020123 exerts oncogenic properties through suppression of miR-144 in non-small cell lung cancer. Am J Cancer Res 2018; 8: 1387-1402.

[15] KROLJ,LOEDIGEI, FILIPOWICZW.The widespread regulation of microRNA biogenesis, function and decay. Nature Rev Genet 2010; 11: 597-610. https://doi.org/10.1038/nrg2843

[16] RUPAIMOOLE R, SLACK FJ. MicroRNA therapeutics: towards a new era for the management of cancer and other diseases. Nat Rev Drug Discov 2017; 16: 203-222. https://doi. org/10.1038/nrd.2016.246

[17] HAN M, WANG S, FRITAH S, WANG X, ZHOU W et al. Interfering with long non-coding RNA MIR22HG processing inhibits glioblastoma progression through suppression of Wnt/ $\beta$-catenin signalling. Brain 2020; 143: 512-530. https:// doi.org/10.1093/brain/awz406

[18] KHOO CP, ROUBELAKIS MG, SCHRADER JB, TSAKNAKIS G, KONIETZNY R et al. miR-193a-3p interaction with HMGB1 downregulates human endothelial cell proliferation and migration. Mol Carcinog 2017; 7: 44137. https://doi. org/10.1002/mc.22826

[19] PAN Y, HU J, MA J, QI X, ZHOU H et al. MiR-193a-3p and miR-224 mediate renal cell carcinoma progression by targeting alpha-2,3-sialyltransferase IV and the phosphatidylinositol 3 kinase/Akt pathway. Mol Carcinog 2018; 57: 1067-1077. https://doi.org/10.1002/mc.22826

[20] TANG Y, YANG S, WANG M, LIU D, LIU Y et al. Epigenetically altered miR-193a-3p promotes HER2 positive breast cancer aggressiveness by targeting GRB7. Int J Mol Med 2019; 43: 2352-2360. https://doi.org/10.3892/ijmm.2019.4167

[21] YU T, LI J, YAN M, LIU L, LIN H et al. MicroRNA-193a$3 p$ and $-5 p$ suppress the metastasis of human non-small-cell lung cancer by downregulating the ERBB4/PIK3R3/mTOR/ S6K2 signaling pathway. Oncogene 2015; 34: 413-423. https://doi.org/10.1038/onc.2013.574

[22] NIE W, GE HJ, YANG XQ, SUN X, HUANG H et al. LncRNA-UCA1 exerts oncogenic functions in non-small cell lung cancer by targeting miR-193a-3p. Cancer Lett 2016; 371: 99-106. https://doi.org/10.1016/j.canlet.2015.11.024

[23] GANAPATHY-KANNIAPPAN S, GESCHWIND J-FH. Tumor glycolysis as a target for cancer therapy: progress and prospects. Mol Cancer 2013; 12: 152. https://doi. org/10.1186/1476-4598-12-152

[24] GANAPATHY-KANNIAPPAN S. Molecular intricacies of aerobic glycolysis in cancer: current insights into the classic metabolic phenotype. Crit Rev Biochem Mol Biol 2018; 53: 667-682. https://doi.org/10.1080/10409238.2018.1556578

[25] JIANG MM, MAI ZT, WAN SZ, CHI YM, ZHANG X et al. Microarray profiles reveal that circular RNA hsa circ_0007385 functions as an oncogene in non-small cell lung cancer tumorigenesis. J Cancer Res Clin Oncol 2018; 144: 667-674. https://doi.org/10.1007/s00432-017-2576-2 
[26] KLIONSKY DJ. Why do we need to regulate autophagy (and how can we do it)? A cartoon depiction. Autophagy 2018; 14 : 1661-1664. https://doi.org/10.1080/15548627.2018.1511218

[27] SÁNCHEZ-MARTÍN P, KOMATSU M. p62/SQSTM1 steering the cell through health and disease. J Cell Sci 2018; 131. https://doi.org/10.1242/jcs.222836

[28] KANG R, ZEH HJ, LOTZE MT, TANG D. The Beclin 1 network regulates autophagy and apoptosis. Cell Death Differ 2011; 18: 571-580. https://doi.org/10.1038/cdd.2010.191

[29] SINGH SS, VATS S, CHIA AY-Q, TAN TZ, DENG S et al. Dual role of autophagy in hallmarks of cancer. Oncogene 2018; 37: 1142-1158. https://doi.org/10.1038/s41388-0170046-6

[30] CHEN P, CESCON M, BONALDO P. Autophagy-mediated regulation of macrophages and its applications for cancer. Autophagy 2014; 10: 192-200. https://doi.org/10.4161/ auto. 26927

[31] KONG R. Circular RNA hsa_circ_0085131 is involved in cisplatin-resistance of non-small-cell lung cancer cells by regulating autophagy. Cell Biol Int 2020; 44: 1945-1956. https:// doi.org/10.1002/cbin.11401

[32] CHEN X, MAO R, SU W, YANG X, GENG Q et al. Circular RNA modulates autophagy via -STAT3-PRKAA/AMPKa signaling in STK11 mutant lung cancer. Autophagy 2020; 16: 659-671. https://doi.org/10.1080/15548627.2019.1634945

[33] BI R, WEI W, LU Y, HU F, YANG X et al. High hsa_ circ_0020123 expression indicates poor progression to nonsmall cell lung cancer by regulating the miR-495/HOXC9 axis. Aging (Albany NY) 2020; 12: 17343-17352. https://doi. org/10.18632/aging.103722

[34] WANG L, ZHAO L, WANG Y. Circular RNA circ_0020123 promotes non-small cell lung cancer progression by sponging miR-590-5p to regulate THBS2. Cancer Cell Int 2020; 20: 387. https://doi.org/10.1186/s12935-020-01444-Z
[35] XIA T, PAN Z, ZHANG J. CircPDZD8 promotes gastric cancer progression by regulating CHD9 via sponging miR-1975p. Aging (Albany NY) 2020; 12: 19352-19364. https://doi. org/10.18632/aging.103805

[36] LIN M, ZHANG Z, GAO M, YU H, SHENG H et al. MicroRNA-193a-3p suppresses the colorectal cancer cell proliferation and progression through downregulating the PLAU expression. Cancer Manag Res 2019; 11: 5353-5363. https:// doi.org/10.2147/CMAR.S208233

[37] LIU Y, XU X, XU X, LI S, LIANG Z et al. MicroRNA-193a$3 p$ inhibits cell proliferation in prostate cancer by targeting cyclin D1. Oncol Lett 2017; 14: 5121-5128. https://doi. org/10.3892/ol.2017.6865

[38] LIU X, MIN S, WU N, LIU H, WANG T et al. miR-193a$3 p$ inhibition of the Slug activator PAK4 suppresses nonsmall cell lung cancer aggressiveness via the p53/Slug/ L1CAM pathway. Cancer Lett 2019; 447: 56-65. https://doi. org/10.1016/j.canlet.2019.01.027

[39] TANIGUCHI T, OGASAWARA K, TAKAOKA A, TANAKA N. IRF family of transcription factors as regulators of host defense. Annu Rev Immunol 2001; 19: 623-655. https://doi. org/10.1146/annurev.immunol.19.1.623

[40] WANG L, NING S. Interferon regulatory factor 4 is activated through c-Src-mediated tyrosine phosphorylation in virustransformed cells. J Virol 2013; 87: 9672-9679. https://doi. org/10.1128/JVI.01435-13

[41] WEI CX, WONG H, XU F, LIU Z, RAN L et al. IRF4-induced upregulation of IncRNA SOX2-OT promotes cell proliferation and metastasis in cholangiocarcinoma by regulating SOX2 and PI3K/AKT signaling. Eur Rev Med Pharmacol Sci 2018; 22: 8169-8178. https://doi.org/10.26355/ eurrev_201812_16509

[42] QIAN Y, DU Z, XING Y, ZHOU T, CHEN T et al. Interferon regulatory factor 4 (IRF4) is overexpressed in human non-small cell lung cancer (NSCLC) and activates the Notch signaling pathway. Mol Med Rep 2017; 16: 6034-6040. https://doi.org/10.3892/mmr.2017.7319 
https://doi.org/10.4149/neo_2022_211013N1449

Circ_0020123 regulates autophagy, glycolysis, and malignancy by upregulating IRF4 through eliminating miR-193a-3p-mediated suppression of IRF4 in non-small cell lung cancer

Xing-Ping YANG ${ }^{1, *}$, Yu-Zhen ZHENG ${ }^{1, *}$, Jian TAN ${ }^{1}$, Ren-Jiang TIAN², Piao SHEN ${ }^{2}$, Wei-Jie CAI ${ }^{1}$, Hong-Ying LIAO ${ }^{1, *}$

Supplementary Information



Supplementary Figure S1. RNA pull-down assay was performed to further validate the relationship between circ_0020123 and miR-193a-3p. ${ }^{* * *} \mathbf{p}<0.001$ 